\title{
Assessment of a dynamic reference material for calibration of full-field measurement systems
}

\author{
Erwin Hack ${ }^{\text {la }}$, Mara Feligiotti ${ }^{\mathrm{a}}$, Andrea Davighi ${ }^{\mathrm{b}}$, Maurice Whelan ${ }^{\mathrm{b}}$, Weizhuo Victor Wang ${ }^{\mathrm{c}}$, \\ Eann A. Patterson ${ }^{c}$ \\ ${ }^{a}$ Electronics/Metrology/Reliability Laboratory, Empa, Ueberlandstrasse 129, \\ CH-8600 Duebendorf, Switzerland, \\ ${ }^{\mathrm{b}}$ Institute for Health and Consumer Protection, European Commission DG Joint Research Centre, \\ 21027, Ispra (VA), Italy \\ ${ }^{c}$ School of Engineering, University of Liverpool, The Quadrangle, Liverpool L69 3GH, UK
}

\begin{abstract}
For holography and speckle interferometry the calibration of the sensitivity is a must, because illumination and observation directions vary across the field of view. A numerical estimate or a static calibration using rigid body motions is standard, and reference materials exist for static strain calibration. Recently, reference materials for the dynamic calibration of optical instruments of displacement and strain measurement were designed and prototypes were manufactured in the European FP7 project ADVISE. We review the properties of the reference material and the concept of traceability for the field of displacement values by using a calibrated single point transducer. The mode shape is assessed using out-of-plane DSPI, Finite Element Analysis as well as analytic solutions of the plate vibration. We present measurements using stroboscopic DSPI on the reference material under acoustic excitation and compare the measured mode shapes to the ones predicted by FE analysis. We apply different comparison methodologies based on point-by-point deviations and on decomposition of the mode shapes into a set of orthogonal basis functions. The latter method is well suited to assess stability and reproducibility of a mode shape. Finally, the deviations are used to estimate the reference material uncertainty which is an essential parameter for determining the calibration uncertainty. Uncertainty contributions of the DSPI set-up are taken into account. To conclude, the application area and limitations of the reference material are discussed.
\end{abstract}

Keywords: reference material, calibration, traceability, DSPI, holography, image correlation.

\section{INTRODUCTION}

Laser speckle interferometry is used for the measurement of displacement and strain fields. While the calibration of the wavelength of the laser is normally not necessary, the calibration of the in- and out-of plane sensitivity is a must, because the illumination and observation directions vary across the field of view and depend on object shape. A numerical estimate or a static calibration using e.g. rotations or translations is standard, and reference materials exist for static strain calibration from the European SPOTS project [1]. For dynamic processes, most notably for full-field measurements, there is a lack of accepted calibration methodologies. To overcome this lack, a reference material for the dynamic calibration of optical methods of displacement and strain measurement was designed and manufactured in the European FP7 project ADVISE [2].

\footnotetext{
${ }^{1}$ Corresponding author: erwin.hack@empa.ch
}

Speckle 2012: V International Conference on Speckle Metrology, edited by

Ángel F. Doval, Cristina Trillo, J. Carlos López-Vázquez, Proc. of SPIE Vol. 8413, $84130 \mathrm{U}$

(c) 2012 SPIE · CCC code: $0277-786 X / 12 / \$ 18 \cdot$ doi: $10.1117 / 12.977950$ 


\section{DYNAMIC REFERENCE MATERIAL}

The dynamic reference material can be used for all kind of contactless optical measurement techniques including DSPI, digital image correlation, or holography. The design and manufacturing processes for the reference material have been described elsewhere [3][4]. It consists of a rectangular plate that is machined from a massive frame. Hinges at the corners remain to bridge the plate to the frame (Fig. 1). Free edges were chosen to enhance the out-of-plane amplitude compared to a plate clamped all around its circumference. This monolithic design guarantees stable boundary conditions which are somewhere between simply-supported and clamped corners. Further, the design is parametric and based on the longitudinal dimension $a$ of the rectangular membrane. Its width is $b=2 a / 3$ and the thickness of the membrane, $t$, is specified as a fraction of the length. The prototype was milled from cast aluminum alloy 5083 (Peraluman). Care was taken to avoid residual stresses in the machining process. The manufactured reference material has dimensions, $a=105 \mathrm{~mm}, b=70 \mathrm{~mm}$ and thickness $t=1 \mathrm{~mm}$. By design, the resonances are well separated in frequency, because the stability and reproducibility of the mode shapes is essential for a reference material, and mode-mixing should be avoided. The plate vibrations were assessed using out-of-plane DSPI, Finite Element Analysis, and analytic approximations. Let the $n^{\text {th }}$ mode shape be described by

$$
w_{n}(x, y ; t)=W_{0} f_{n}(x, y) \cos \left(\omega_{n} t\right)
$$

where $f_{n}$ represents the normalized mode function, while $W_{0}$ is the scaling factor which depends on the excitation force.

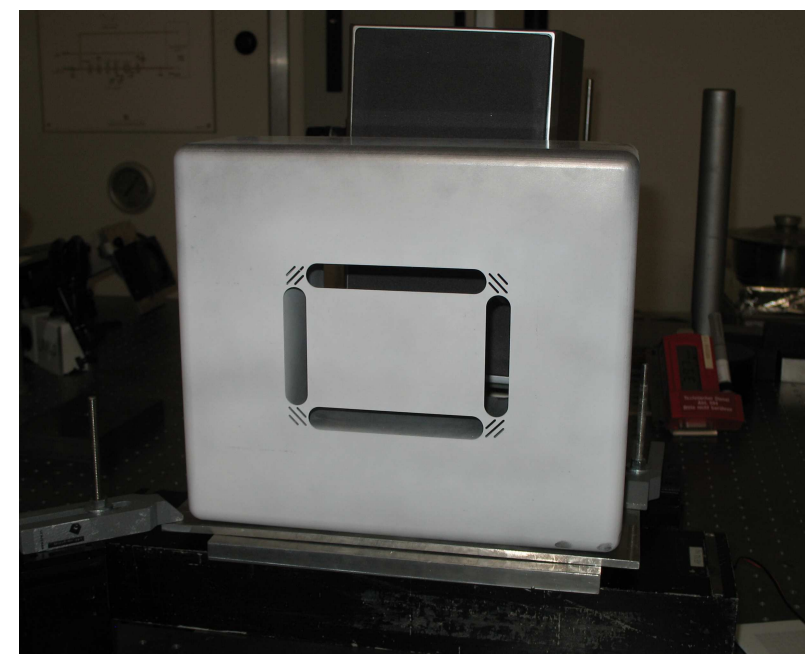

Fig. 1: Reference material with surface powdered for better diffusivity. The loudspeaker used for excitation is visible in the background.

There are analytic solutions for plate vibrations $f_{n}$, assuming ideal dimensions, material properties, excitation and boundary conditions. For a real object, the normalized mode function $f_{n}$ is not known, but can be parameterized to sufficient accuracy using a series expansion

$$
f_{n}(x, y)=\sum_{i, j} a_{i j}^{n} T_{i}(x) T_{j}(y)
$$

The basis functions $T_{i}$ can be sine and cosine-functions or orthogonal polynomials such as Chebyshev's [5]. 


\section{RESULTS}

Measurements using time-average and stroboscopic DSPI were performed on the reference material under acoustic excitation. Because there is a relative phase-shift of the sinusoidal deflection and the exciting force through the resonance by $180^{\circ}$ care must be taken when performing the stroboscopic DSPI to take this shift into account in order to obtain the maximum deflection. Fig. 2 shows a comparison of the first two measured mode shapes to the ones predicted by FE analysis. In contrast to the calibration, for the comparison to FE, the absolute value of the amplitude is unimportant as long as the mode shape function is unchanged. The amplitude can be adjusted by adjusting the excitation force to a signal level appropriate for the measurement technique at hand.
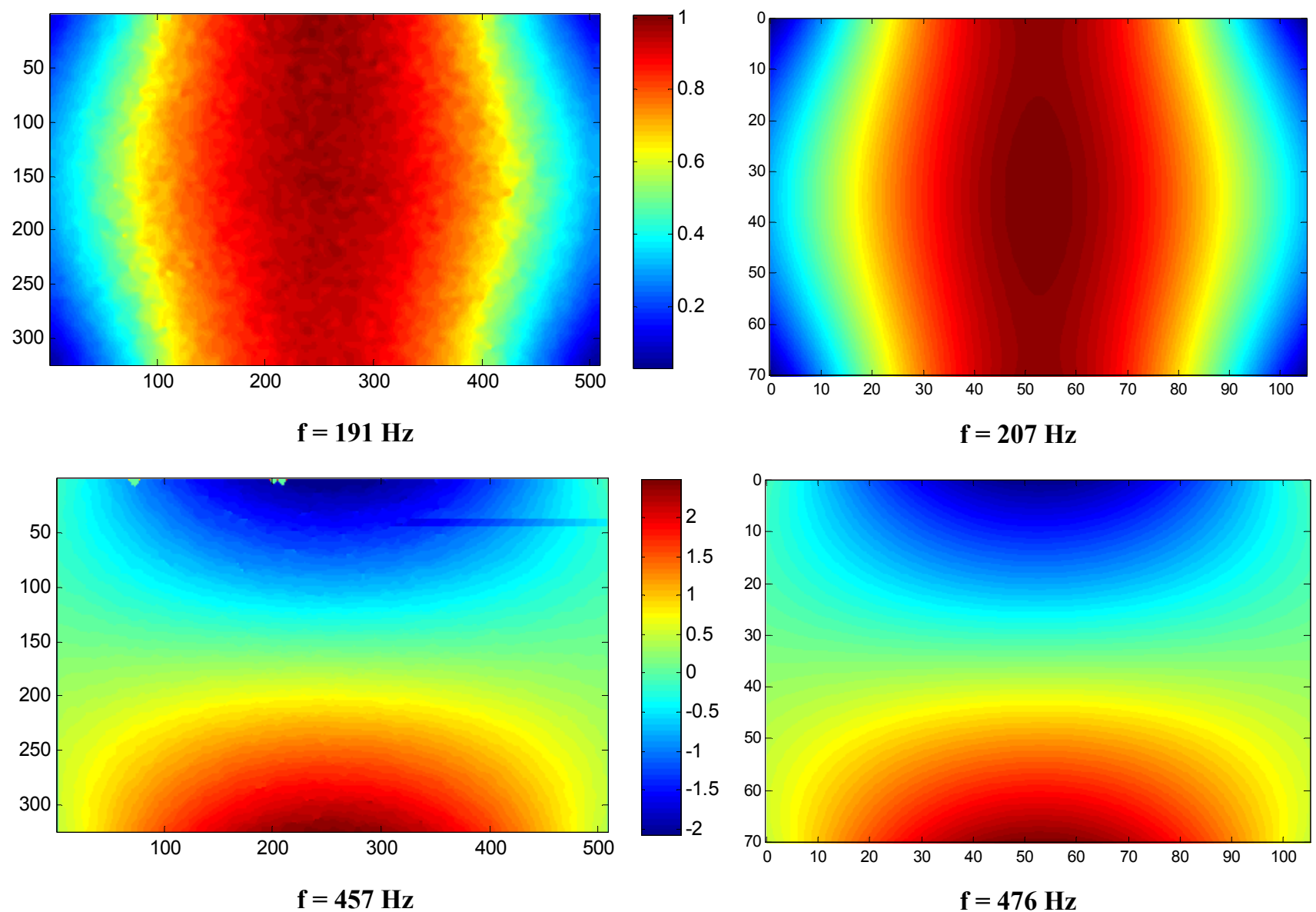

Fig. 2: Resonance frequency and mode shape of the first two resonant modes of the ADVISE reference material. Left: DSPI measurements (axes in pixels); right: FE analysis (axes in $\mathrm{mm}$ ).

The following Fig. 3 (left) shows the resonance curve for the first mode measured by laser Doppler velocimetry. Note that the resonance frequency has shifted by some $20 \mathrm{~Hz}$ compared to Fig. 2. This effect was attributed to a contacting excitation method in a laboratory during a small round robin exercise. Fig. 3 (right) shows the phase lag with a typical jump of pi radians through the resonance frequency. Both curves corroborate the fact that the first mode is a well isolated resonance. 

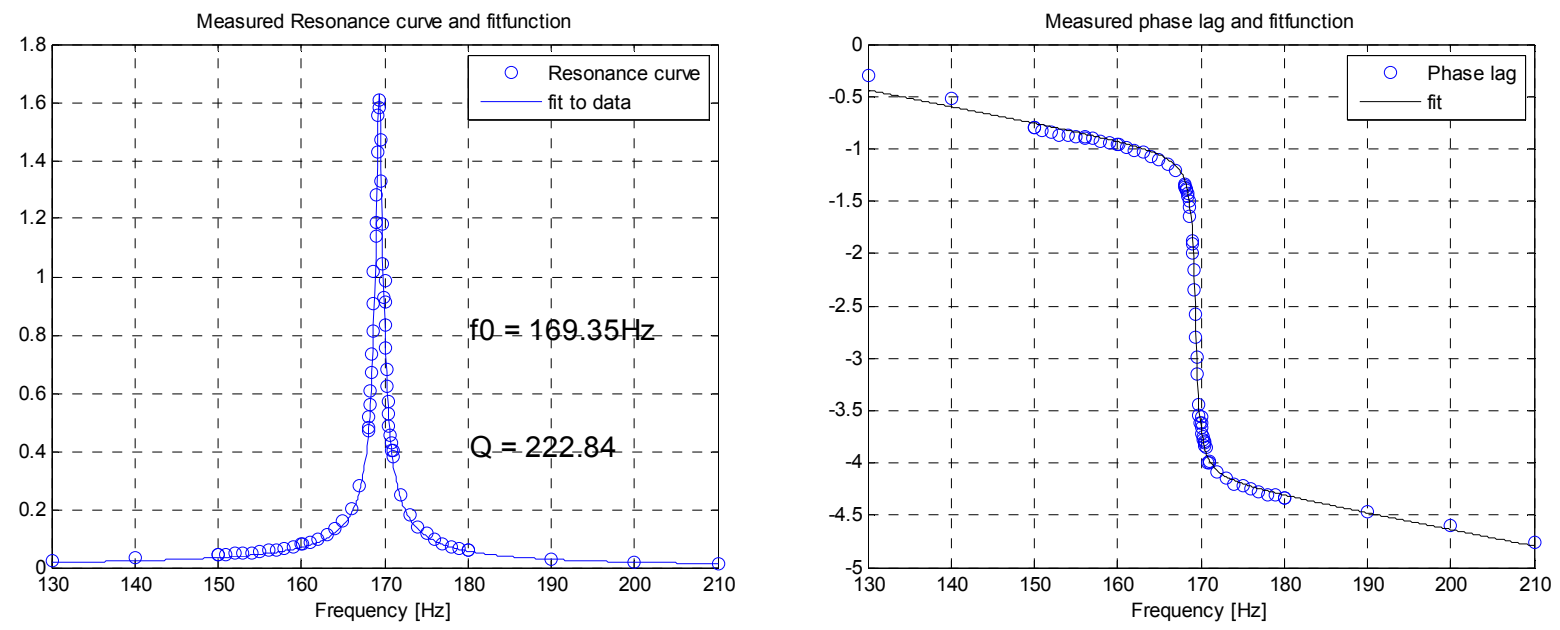

Fig. 3: Resonance curve of the first resonance mode at $169.35 \mathrm{~Hz}$ (left). Phase lag of the surface displacement with respect to the excitation (right).

\section{TRACEABILITY AND CALIBRATION UNCERTAINTY}

Traceability to the unit of length is established for the entire field of reference values by using a single point, noncontacting calibrated transducer such as a laser Doppler vibrometer in selected locations, preferably near the locations of maximum deflection. This is acceptable, since the reference material is operated in its linear regime, i.e. the deflection field $W(x, y)$ scales with $W_{0}$. Using the normalized mode function $f_{n}$, Eq. (1), the local displacement values at each point on the membrane surface can be determined. It is important to synchronize the LVDT and DSPI stroboscope in order to have an exact correspondence of the signals in time.

Contributions to the uncertainty of the reference values $w(\mathrm{x}, \mathrm{y})$ include effects by (i) geometry (manufacturing tolerances) (ii) material (homogeneity) (iii) boundary conditions (symmetry of four corners), (iv) excitation (load distribution on plate) (v) temperature (heating of material, frequency) (vi) frequency (stability) (vii) timing (synchronization of DSPI and LVDT) (viii) measurement uncertainty of LVDT. These contributions are not independent, and therefore should not be quantified individually, unless a model for the calculation of the covariances is available.

It is more convenient to start from Eq.(1) and assign the uncertainty contributions to the independent variables, i.e. amplitude $W_{0}$, mode shape $f_{n}$, and frequency $\omega_{n}$. The contributions (i) through (iv) listed above affect the mode shape function; (v) and (vi) the frequency, while (vii) and (viii) influence the amplitude. From Eq.(1)

$$
\begin{gathered}
u^{2}\left(w_{n}\right)=u^{2}\left(W_{0}\right) f_{n}^{2}(x, y) \cos ^{2}\left(\omega_{n} t\right)+W_{0}{ }^{2} u^{2}\left(f_{n}(x, y)\right) \cos ^{2}\left(\omega_{n} t\right)+ \\
W_{0}{ }^{2} f_{n}{ }^{2}(x, y) \sin ^{2}\left(\omega_{n} t\right)\left[t^{2} u^{2}\left(\omega_{n}\right)+\omega_{n}{ }^{2} u^{2}(t)\right]
\end{gathered}
$$

Since we assume that the DSPI stroboscope and the laser vibrometer are synchronized to the driving force, the last term is not representative for the frequency uncertainty. Rather, a jitter in frequency should be assumed. The uncertainty in amplitude is detailed as follows:

$$
u^{2}\left(W_{0}\right)=u^{2}(L V D T)+u^{2}(\text { position })+u^{2}(\text { repetition })
$$


where $u(L V D T)$ is the measurement uncertainty of the LVDT, $u$ (position) reflects the uncertainty in pointing the LVDT to the prescribed location of maximum deflect ion; and $u$ (repetition) is the sine-to-sine change of the resonant vibration. The uncertainty in the mode shape can be estimated by the rms deviation from the reconstructed mode shape to the simulated one.

For the calibration uncertainty of the DSPI instrument, the speckle noise dominates the reconstruction uncertainty, i.e. expansion coefficients lower than the speckle noise level are insignificant. In Fig. 4 (left) the residual after reconstruction using 16x16 orders is given, while Fig. 4 (right) shows the corresponding rms values for the residuals when reconstruction is performed using the coefficients in descending order of their magnitude. It is seen that the DSPI reconstruction levels off at the DSPI noise of around $8 \mathrm{~nm}$ (depending on filtering strength applied to the phase map), while the smooth FEA result has a limiting value of zero. In both cases, the reconstruction using the 20dominant terms is of sufficient quality. From this result, the contribution of the speckle noise to the DSPI calibration uncertainty can be approximated by $8 \mathrm{~nm}$.
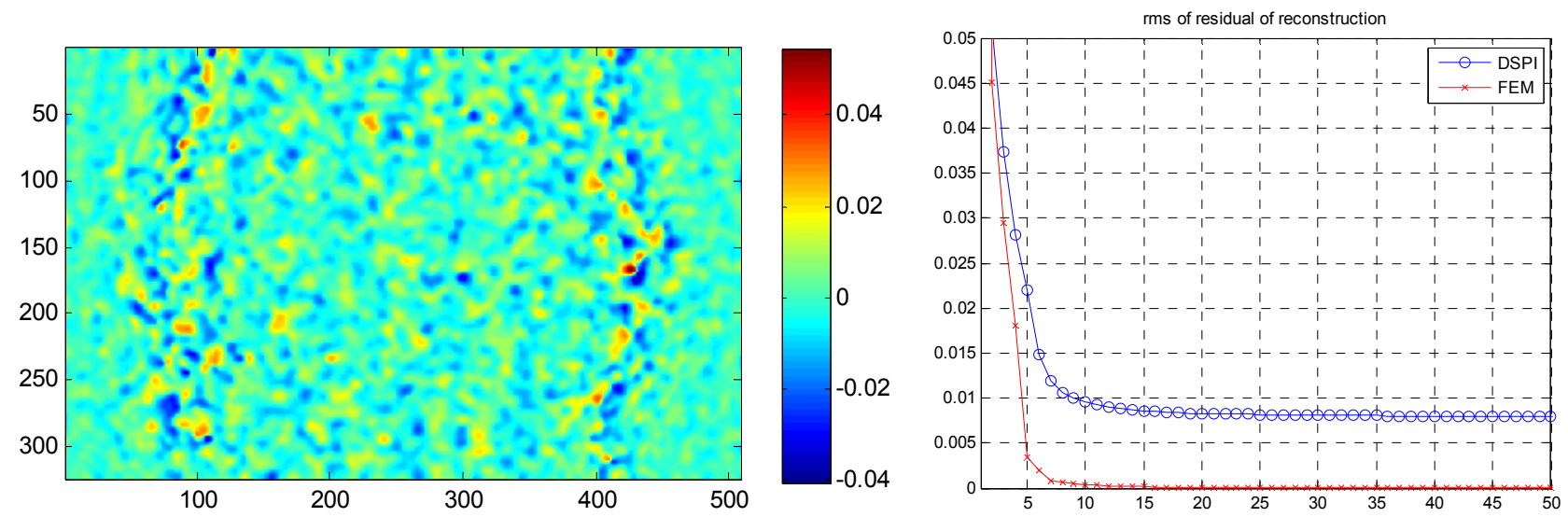

Fig. 4: Residual difference $[\mu \mathrm{m}]$ between the first mode shape measured by DSPI and the reconstruction using Chebyshev polynomials. Left: full-field-difference; right: rms value for reconstruction of DSPI and FEM results

In addition, stroboscopic DSPI uses a finite integration time over each cycle. This implies that the measured displacement value has slightly lower amplitude than the maximum amplitude incorporated in the reference material.

\section{CONCLUSIONS}

The reference material as described in this paper is an artifact for calibrating measurement systems for dynamic deformations. Its mode shape can be parameterized based on orthogonal functions, and the uncertainty of the reference values was estimated. An application to the calibration of a DSPI instrument was sketched It is expected that the dynamic reference material will be made available to the interested for further testing using round robins in the frame work of the European CSA project VANESSA in 2013. 


\section{ACKNOWLEDGMENTS}

The authors gratefully acknowledge contributions from members of the EU FP7 project ADVISE consortium which was funded by the European Commission through Grant no. 218595.

\section{REFERENCES}

[1] SPOTS (Standardisation Project for Optical Techniques of Strain Measurement), see www.opticalstrain.org

[2] ADVISE (Advanced Dynamic Validation using Integrated Simulations and Experimentation), Grant Agreement No. SCP7-GA-2008-218595, see www.dynamicvalidation.org

[3] A. Davighi, R. Burguete, M. Feligiotti, E. Hack, S. James, E. Patterson, The development of a reference material for calibration of full-field optical measurement systems for dynamic deformation measurements, Applied Mechanics and Materials 70 (2011) 33-38

[4] A. Davighi, M. Feligiotti, E. Hack, E. Patterson, M. Whelan, Design of a reference material for the calibration of modal displacement measurements, Exp. Tech., submitted

[5] see e.g. M. Abramowitz, I.A. Stegun, Eds., Handbook of mathematical functions, Courier Dover Publications, 1964

[6] W. Wang, J.E. Mottershead, C. Sebastian, E.A. Patterson, Th. Siebert, A. Ihle; A. Pipino, Image Analysis for Full-Field Displacement/Strain Data: Method and Applications, Applied Mechanics and Materials 70 (2011) 39-44 